\title{
Etude des variations spatio-temporelles de la pollution des eaux de la lagune de Porto-Novo (sud Bénin)
}

\author{
Waris CHOUTI ${ }^{1,2^{*}}$, Daouda MAMA ${ }^{1,2}$ et François ALAPINI ${ }^{1}$ \\ ${ }^{1}$ Laboratoire de Chimie Minérale, Faculté des Sciences et Techniques (FAST), Université d'Abomey-Calavi, \\ BP : 4521 Cotonou Bénin. \\ ${ }^{2}$ Laboratoire d'Hydrologie Appliquée, Faculté des Sciences et Techniques (FAST), Université d'Abomey- \\ Calavi, 01 BP : 526 Cotonou Bénin. \\ *Auteur correspondant, E-mail : lawaniwaris@yahoo.fr; Tel :00229 97487320
}

\section{RESUME}

La lagune de Porto-Novo, d'une superficie de $50 \mathrm{~km}^{2}$, est située au sud-est du Bénin, et constitue l'exutoire par lequel les eaux du fleuve Ouémé se jettent dans l'océan Atlantique par le chenal de Lagos. Elle est à l'image de ces cours d'eau, lacs et lagunes de certaines grandes villes de l'Afrique de l'Ouest dans lesquels se déversent presque toutes les eaux usées de la ville. Les eaux de six points de cette lagune ont été prélevées et analysées, pendant les quatre saisons de l'année, afin de déterminer les teneurs en fer, manganèse, chrome, plomb, cadmium et mercure. Les métaux ( $\mathrm{Fe}, \mathrm{Cd}, \mathrm{Pb}, \mathrm{Cr}, \mathrm{Mn}$ ) ont été déterminés par la méthode des kits MERCK au spectrophotomètre NOVA 60. Le mercure a été déterminé d'après la méthode de préconcentration à la vapeur froide suivie d'un dosage colorimétrique au spectrophotomètre HACH DR2800. Le traitement des données a été fait avec le logiciel SPSS 12. Les résultats ont montré que les valeurs annuelles des teneurs des eaux des points étudiés dépassent les valeurs seuil, pour les différents métaux, recommandées par le Conseil Canadien des Ministres de l'Environnement. Les résultats font remarquer que les eaux de ruissellement constituent une source de pollution non négligeable.

(C) 2010 International Formulae Group. All rights reserved.

Mots-clés : paramètres physicochimiques, pollution métallique, mercure, plomb, fer, chrome, Bénin.

\section{INTRODUCTION}

L'expansion démographique dans les pays en voie de développement s'accompagne d'un autre problème qui est souvent mal géré : la gestion des déchets solides et liquides. Ces déchets continuent d'être rejetés sans traitement dans les cours d'eau, lacs et lagunes de certaines grandes villes de l'Afrique de l'Ouest, posant de graves problèmes de santé (Evaluation des progrès du développement durable en Afrique depuis Rio, 2001 ; Lamizana-Diallo et al., 2008). De plus, la contamination des écosystèmes aquatiques par les métaux demeure-t-elle un sérieux problème d'environnement de plus en plus inquiétant (Reyms-Keller et al., 1998). Ces métaux sont présents dans tous les compartiments de l'écosystème aquatique (eau, sédiment, faune et flore)(Langstone, 1999). Les mesures effectuées sur les eaux 
d'un cours d'eau donnent une indication sur la pollution $\mathrm{du}$ milieu au moment du prélèvement (Boucheseche, 2002).

En absence de traitement et du manque de contrôle des eaux de ruissellement qui finissent par s'y retrouver, la composition chimique notamment métallique de ces cours d'eau peut varier fortement selon les différentes saisons de l'année et se détériorer au cours des années.

L'étude des variations spatiotemporelles de la composition métallique des cours d'eau permet non seulement de caractériser ces polluants qui peuvent être à l'origine de la disparition de certaines espèces animales et/ou végétales et par conséquent, entraîner le dysfonctionnement de la chaîne trophique (Bloundi, 2005). Elle permet aussi de connaitre les sites les plus pollués et les périodes de l'année où la pollution est plus accentuée.

Les rares études de pollution chimique effectuées sur la lagune de Porto-Novo (sud Bénin) n'ont été que ponctuelles. Or cette lagune reçoit régulièrement les déchets liquides domestiques des populations par l'intermédiaire des collecteurs d'eau qui se retrouvent le long de la lagune.

La présente étude vise à cerner les variations saisonnières en métaux lourds de la lagune, à connaître les sites les plus pollués et à comprendre les causes de la mort des poissons souvent signalée par les pêcheurs.

\section{MATERIEL ET METHODES}

\section{Prélèvement des échantillons}

Six points de prélèvements ont été choisis en tenant compte des déversements domestiques, eaux de ruissellement et apport du Lac Nokoué (Figure 1). Les campagnes de prélèvements sont effectuées suivant les quatre saisons de l'année: le 29 novembre 2008 (petite saison pluvieuse), le 07 mars 2009 (grande saison sèche), 29 juin 2009 (début de la grande saison des pluies) et 07 septembre 2009 (fin de la grande saison des pluies qui correspond à la période des grandes eaux de la lagune).

L'échantillonnage a été réalisé comme suit :

- Les bouteilles utilisées pour les prélèvements d'eau sont préalablement lavées et rincées au laboratoire, rincées avec l'eau à prélever sur le terrain.

- Le prélèvement d'eau est effectué à environ $5 \mathrm{~cm}$ de la surface.

- Les bouteilles remplies complètement sont fermées hermétiquement pour éviter toute fuite de gaz.

\section{Produits, matériels et méthodes}

Les eaux analysées ont été prélevées aux six points cités précédemment de la lagune. Les eaux ont été prélevées dans des bouteilles en plastique. Elles ont été minéralisées selon la méthode Digesdahl Hach avant l'analyse des métaux. Le pH et l'oxygène dissous ont été mesurés par pH/Oxymétrie avec une sonde WTW 340i. La température, la salinité, la conductivité et le TDS par conductimétrie avec une sonde WTW 340i.

La turbidité, les MES (Matières En Suspension) et la couleur ont été mesurés grâce à un colorimètre HACH DR890. Les métaux ( $\mathrm{Fe}, \mathrm{Cd}, \mathrm{Pb}, \mathrm{Cr}, \mathrm{Mn}$ ) ont été déterminés par la méthode des kits MERCK au spectrophotomètre NOVA 60.

Le mercure a été déterminé d'après la méthode de préconcentration à la vapeur froide suivie d'un dosage colorimétrique au spectrophotomètre HACH DR2800.

\section{RESULTATS}

\section{Paramètres physico-chimiques des eaux}

La Figure 2 présente les résultats physicochimiques obtenus durant les quatre campagnes. On constate que ce n'est que pendant la période de basses eaux que les eaux sont salées, la lagune reçoit alors les eaux marines par l'intermédiaire du lac Nokoué. Pendant cette période, la salinité est nulle au point E2 alors qu'elle varie de 3,6\%o 
à $15,5 \%$ au niveau des autres points. La conductivité est élevée pendant la période des hautes eaux même au point E2 où elle a été faible au cours des trois premières saisons. Pour toutes les saisons les valeurs les plus élevées de $\mathrm{pH}$ sont observées au point E3 (Canal de Totchè) alors que les valeurs les plus basses sont observées au point E2.

Pour les saisons, la concentration la plus élevée d'oxygène dissous (elle varie de 2,22 à $8,18 \mathrm{mg} / \mathrm{L}$ ) est obtenue au point $\mathrm{E} 3$ alors que la concentration la plus basse est obtenue au point E2 (elle varie de 0,14 à 2,23 $\mathrm{mg} / \mathrm{L}$ ). La période des hautes eaux est la plus pauvre en oxygène dissous suivie de la période des pluies.

On observe la particularité du point E2. Outre la transparence, les valeurs des paramètres sont partout plus basses à ce point. Ces faibles valeurs en général et celles de la salinité et de la conductivité en particulier s'apparentent à celles des eaux souterraines, donc seraient dues à la présence d'une source souterraine qui alimenterait ce point.

Pour l'oxygène dissous, les valeurs moyennes obtenues sont inférieures à la concentration minimale acceptable (Tableau $1)$.

Ordre de gradeur des concentrations des métaux

Les moyennes annuelles de la teneur métallique des eaux des stations étudiées sont indiquées dans le Tableau 2.

La comparaison des moyennes obtenues aux valeurs seuil montre un dépassement pour tous les métaux. La lagune est donc polluée et sa qualité menace la vie aquatique. Lorsqu'on considère les conséquences biologiques néfastes répertoriées pour chaque métal, les conséquences de la mauvaise qualité de ces eaux sur la lagune sont: diminution des invertébrés, accroissement de la mortalité, modifications comportementales, létalité, diminution de la fécondité, développement anormal dans les premiers stades de vie des organismes benthiques.

La mort des poissons souvent constatée au niveau de la lagune peut s'expliquer par la toxicité des eaux engendrée par les teneurs élevées des métaux.

Ces résultats montrent que les valeurs les plus élevées pour le fer et le manganèse sont obtenues au point E4. Ce point était situé non loin d'un important dépôt de déchets d'un marché d'échanges de produits de toutes sortes au bord de la lagune. Mais le point E2 est le plus pollué en mercure.

Tous ces résultats sont les conséquences de la mauvaise gestion des déchets et autres activités humaines qui sont menées au niveau de la lagune.

\section{Les teneurs en fer}

Les variations saisonnières sont les mêmes à tous les points. Les concentrations ont été les plus élevées lors des saisons des pluies et des basses eaux. De plus, pour ces deux périodes, les concentrations les plus élevées sont observées au point E4 (Figure 3). Ce point est non loin d'un dépôt de déchets.

\section{Les teneurs en manganèse}

L'analyse de manganèse n'a pas été effectuée lors de la première campagne. Tout comme pour le fer, les concentrations ont été les plus élevées lors des saisons des pluies et des basses eaux. De plus, les concentrations les plus élevées sont observées au point E4 (Figure 4). Donc, cette pollution par le fer et le manganèse est très problablement liée aux déchets qui se situent le long de la lagune.

\section{Les teneurs en chrome}

Les concentrations ont été les plus élevées lors des saisons des pluies et des basses eaux (Figure 5).

Pour le fer et le chrome, et problablement pour le manganèse, on observe une concentration en saison sèche moins élevée que pendant la saison des pluies. Les eaux de ruissellement, lors des pluies, drainent donc vers la lagune des déchets contenant ces métaux. Cela montre la nécessité de la mise en 
place d'un système de contrôle de toutes les eaux usées y compris les eaux de pluie avant le déversement dans la lagune.

\section{Les teneurs en plomb}

Les concentrations ont été les plus élevées lors des saisons sèches pour les points E2, E3, E5 et E6 (Figure 6). Par contre, pour les points E1 et E4, les concentrations ont été les plus élevées lors de la période de basses eaux. Il faut remarquer que ces deux derniers points sont les points qui subissent plus de pressions humaines.

\section{Les teneurs en cadmium}

Les variations saisonnières sont les mêmes à tous les points. Les concentrations ont été les plus élevées lors de la période de basses eaux (Figure 7). Ceci s'explique par le fait que pendant cette période le volume de l'eau est plus faible donc la concentration plus élevée. Après la période de basses eaux, vient la saison des pluies. Ceci confirme les observations faites pour le fer, le manganèse et le chrome. Les eaux de ruissellement, pendant les pluies, polluent la lagune.

\section{Les teneurs en mercure}

De la saison sèche à la période de basses eaux, la concentration a évolué aux points E1, E3, E4, E5 et E6 (Figure 8). Par contre pour le point E2, la concentration a diminué. Notons que ce point n'est jamais salé même lors de la période de basses eaux où tous les autres points sont salés. De même, ce point a la conductivité et le TDS les plus faibles. Tout ceci permet de supposer la présence d'eaux souterraines à cet endroit qui diluent les eaux.

\section{Relations entre métaux et paramètres physicochimiques}

Le Tableau 3 montre les coefficients de corrélation entre métaux et paramètres physicochimiques. Le coefficient de corrélation détermine la relation entre deux variables et mesure l'intensité de ce lien. Il varie entre -1 et +1 . Lorsqu'il est égal à 1 , il indique un lien parfait entre les variables et lorsqu'il est égal à 0 , il indique une absence de lien. Le signe + signifie que la relation est proportionnelle alors que le signe - signifie que la relation est inversement proportionnelle. Le calcul du coefficient de corrélation permet d'avoir une idée sur les éventuelles relations entre métaux: origine commune, distribution homogène, comportement identique vis-à-vis des processus physicochimiques, fractionnement similaire, influence de l'un sur l'autre.

On note une forte relation entre le chrome, le manganèse et le cadmium. Par contre, le fer et le mercure ne sont pas corrélés. Donc si l'hypothèse des déchets comme origine de contamination du fer est admise alors le mercure ne viendrait pas des déchets mais d'une autre source.

Le manganèse est positivement associé à la température et l'oxygène dissous alors qu'il l'est négativement avec la conductivité, la turbidité et la couleur. On note également qu'aucun métal n'est influencé par le pH.

La turbidité, les MES et la couleur sont fortement liées. Cela se justifie par le fait que les MES sont non seulement responsables de la couleur mais également leur présence trouble l'eau. De même, la conductivité estelle aussi corrélée avec ces trois paramètres. Les MES sont donc des espèces ioniques qui augmentent le pouvoir ionique des eaux de la lagune. Tout ceci justifie la raison pour laquelle la transparence de l'eau est faible lorsque ces quatre paramètres sont élevés (les coefficients de corrélation entre la transparence et chacun des quatre paramètres sont significativement négatifs). On constate également que le $\mathrm{pH}$, l'oxygène dissous et la température sont associés. 
W. CHOUTI et al. / Int. J. Biol. Chem. Sci. 4(4): 1017-1029, 2010

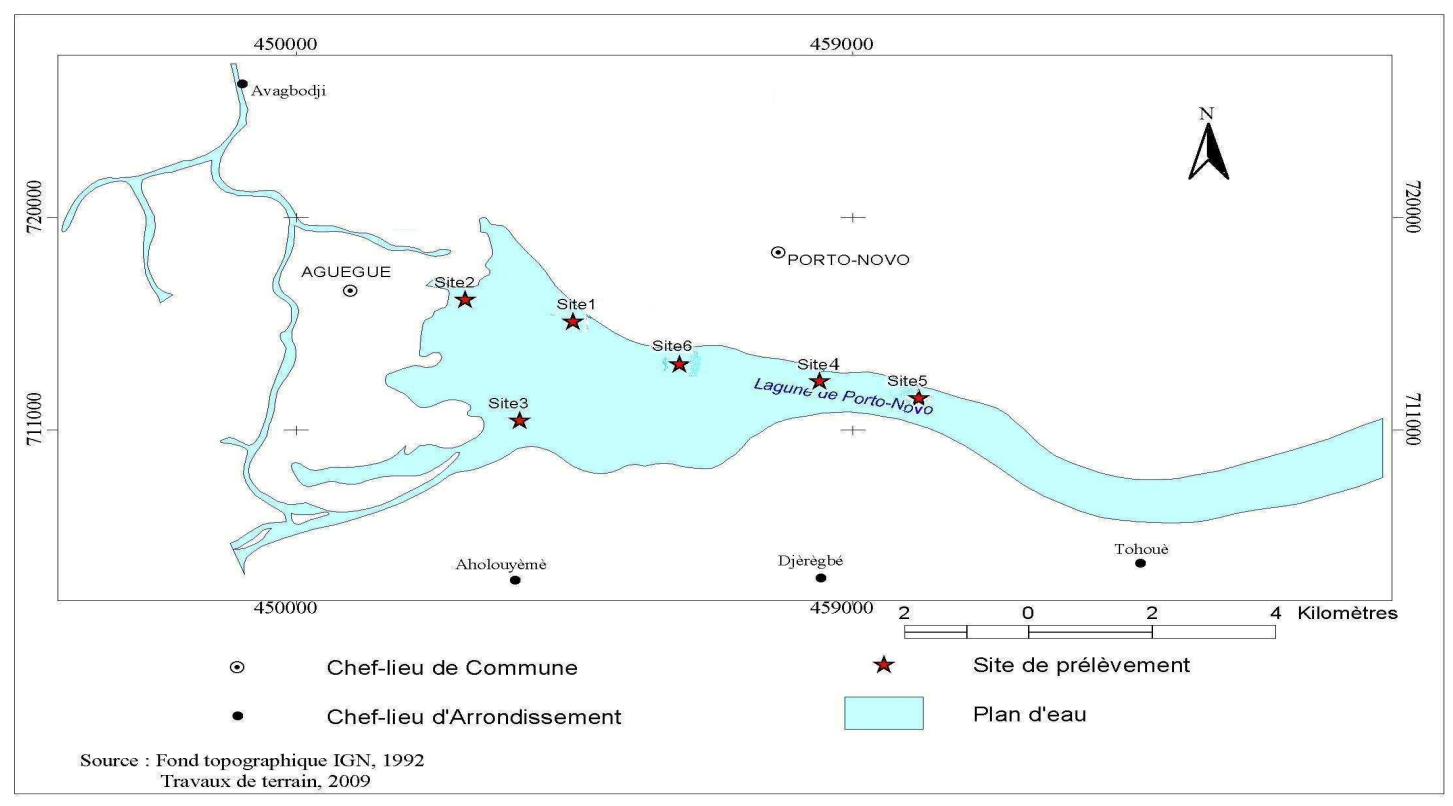

Figure 1: Répartition des sites de prélèvement.
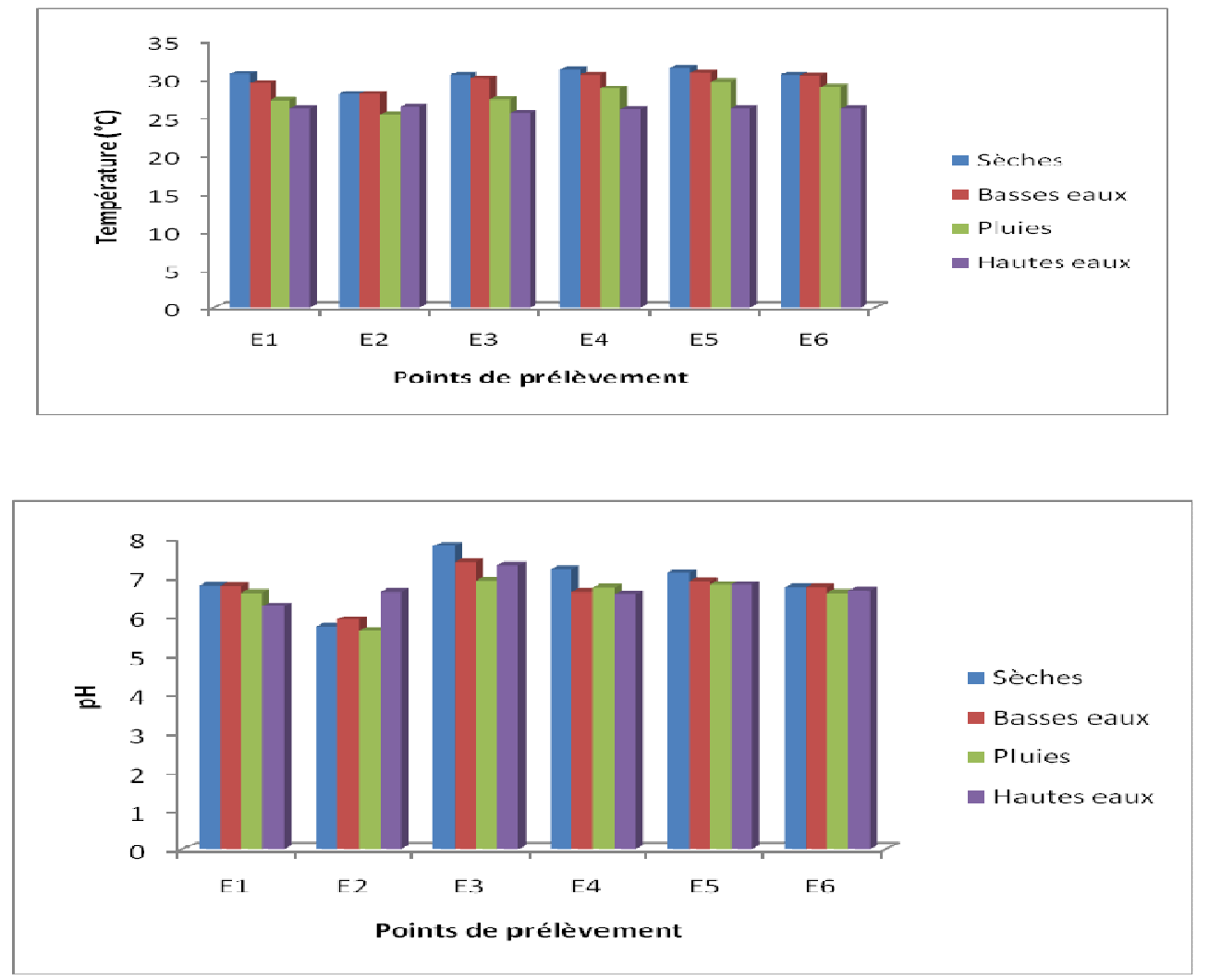
W. CHOUTI et al. / Int. J. Biol. Chem. Sci. 4(4): 1017-1029, 2010
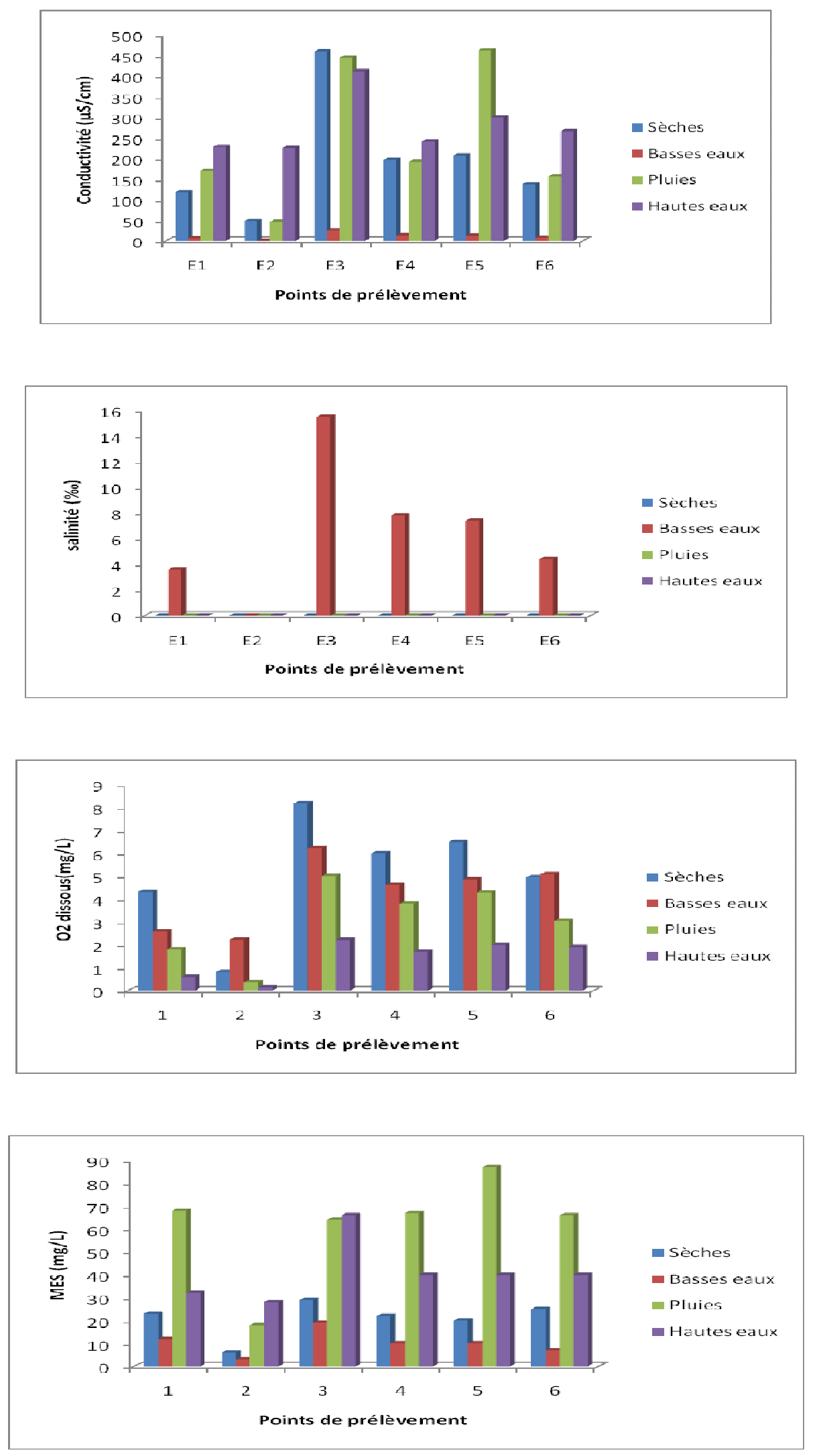

1022 

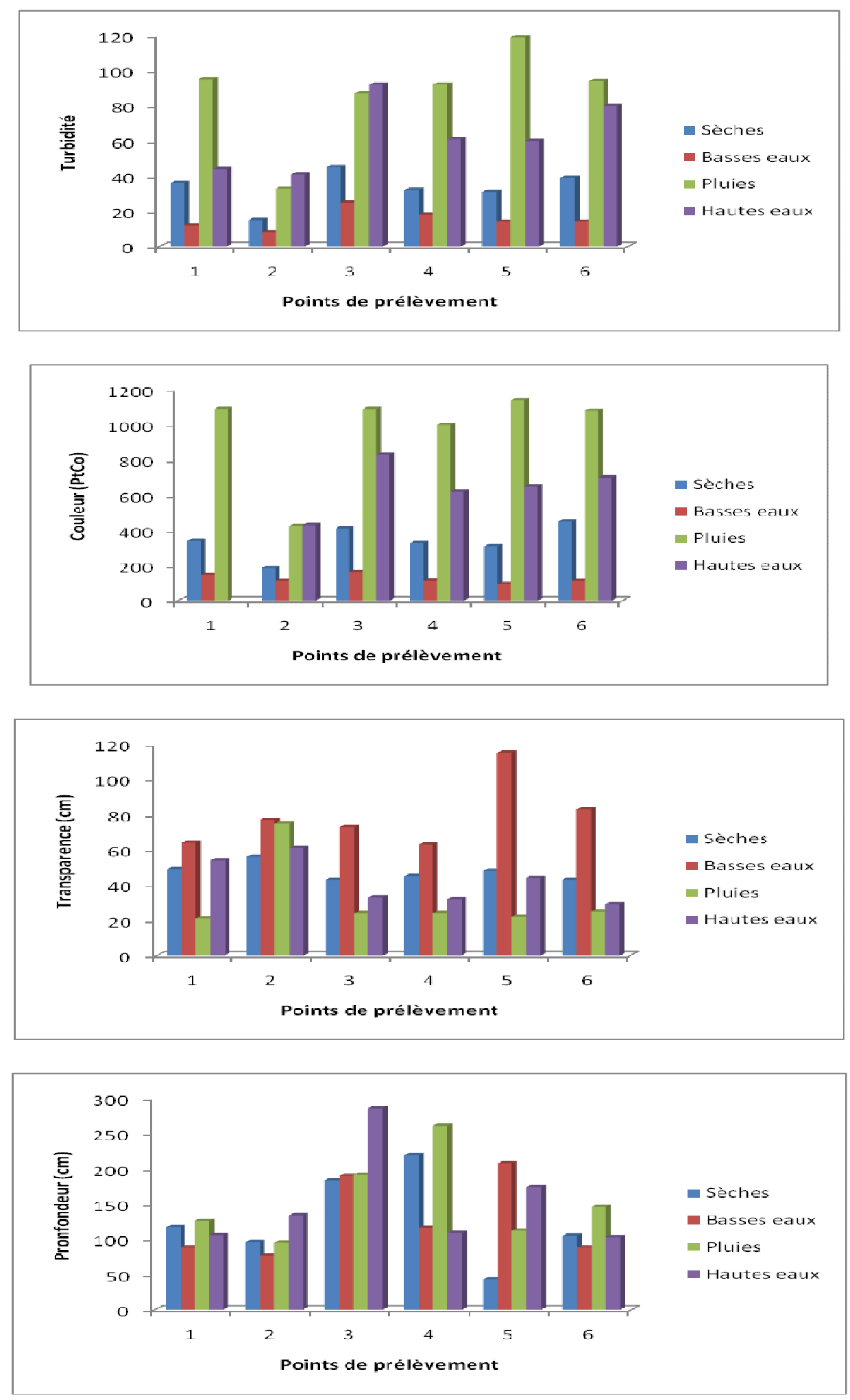

Figure 2 : Variations saisonnières des paramètres physicochimiques de la lagune. 


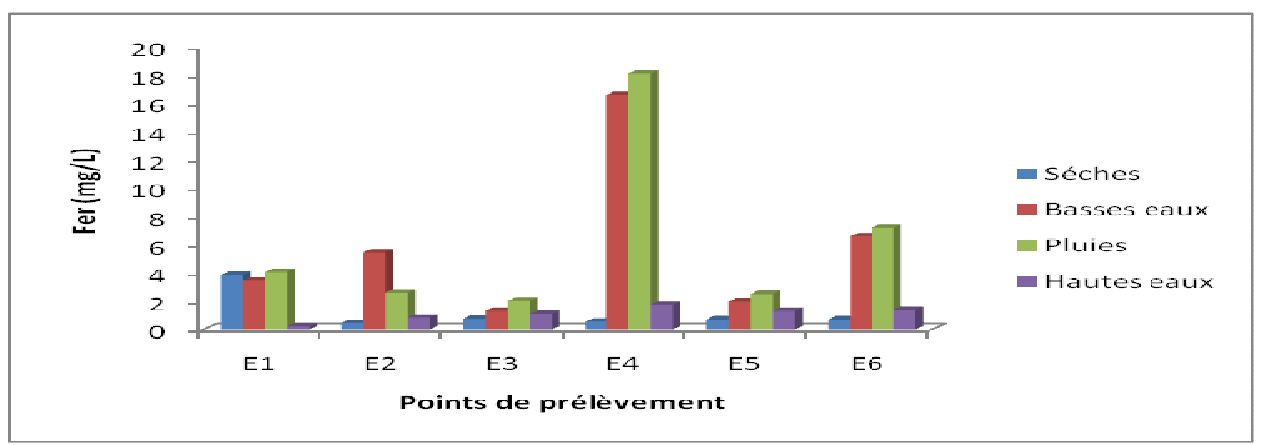

Figure 3 : Variations saisonnières de la teneur en fer des eaux.

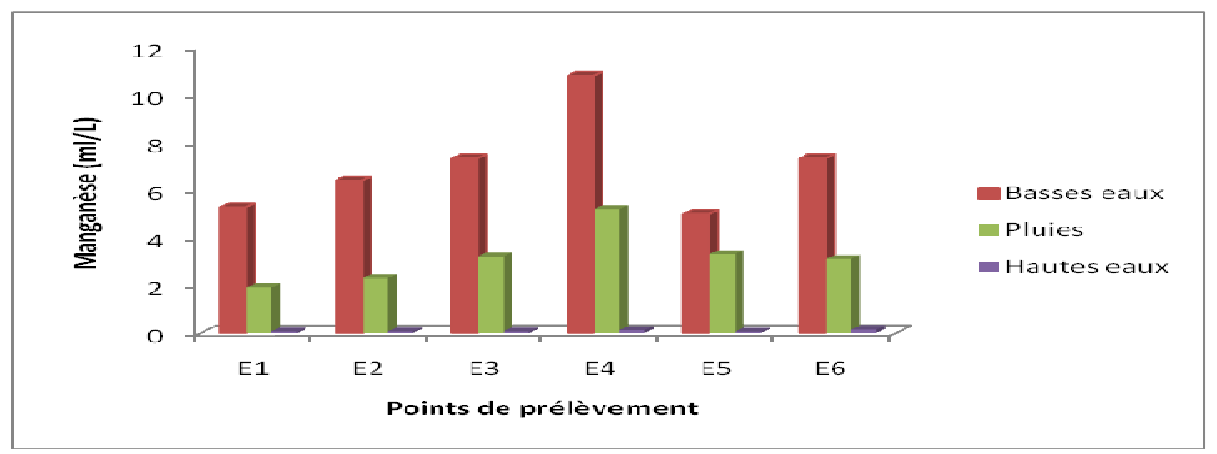

Figure 4 : Variations saisonnières de la teneur en manganèse des eaux.

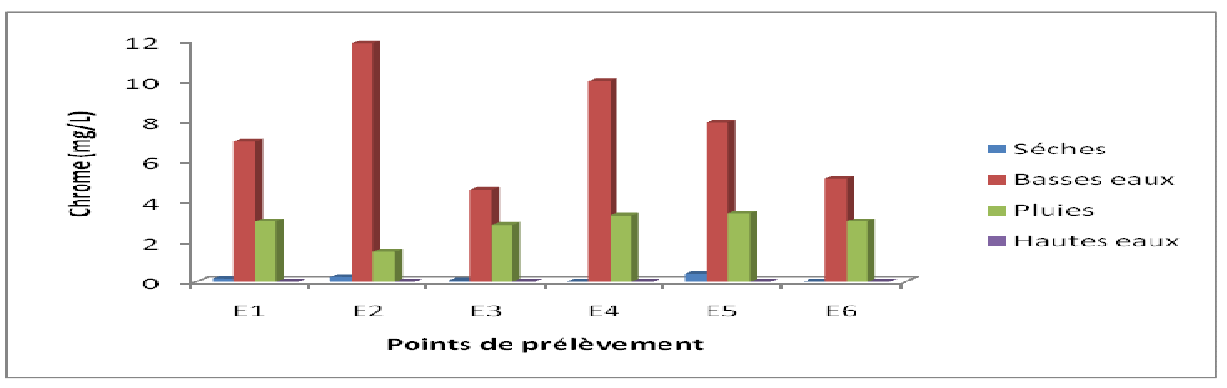

Figure 5 : Variations saisonnières de la teneur en chrome des eaux.

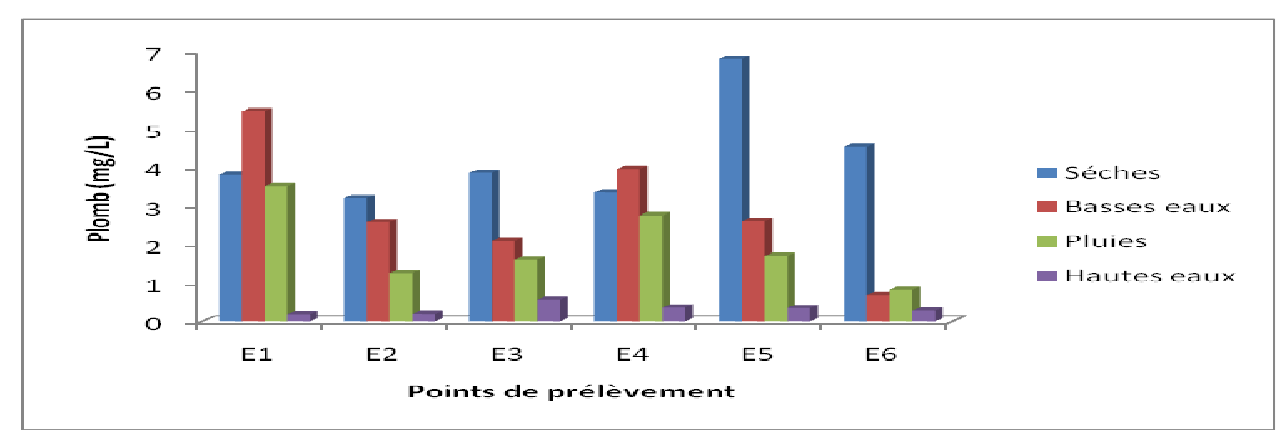

Figure 6 : Variations saisonnières de la teneur en plomb des eaux. 


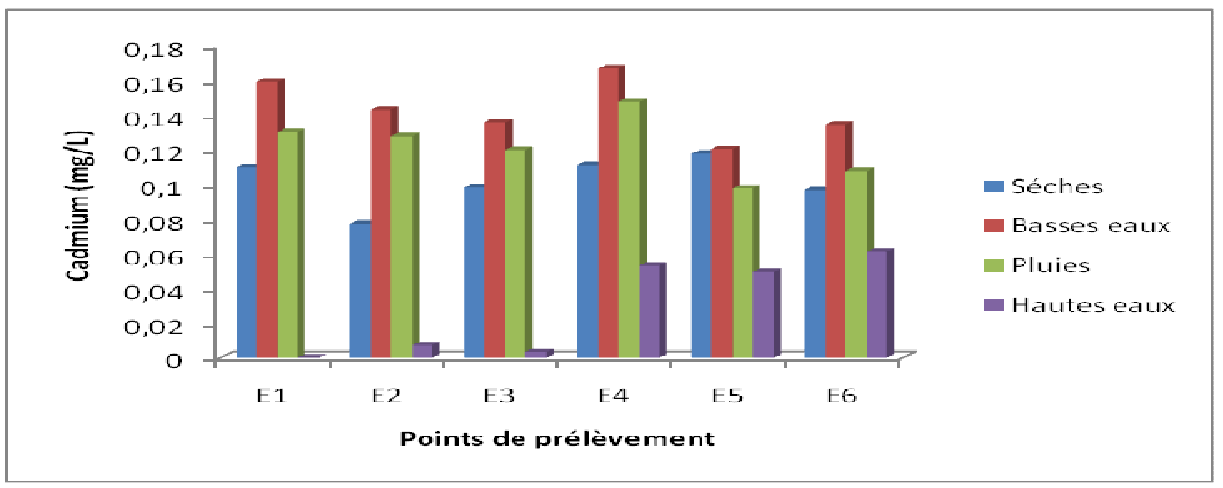

Figure 7 : Variations saisonnières de la teneur en cadmium des eaux.

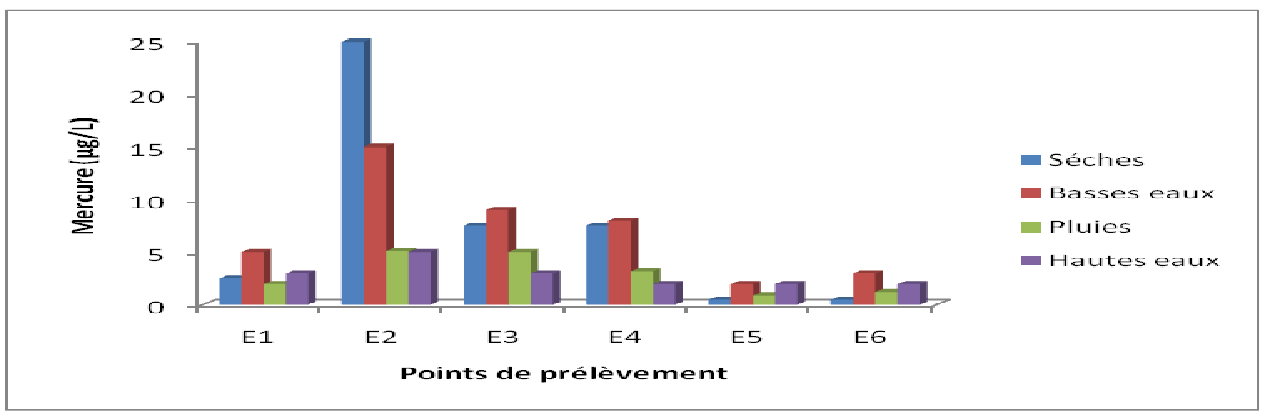

Figure 8 : Variations saisonnières de la teneur en mercure des eaux.

Tableau 1 : Moyennes annuelles de la concentration de l'oxygène dissous.

\begin{tabular}{lccccccc}
\hline & E1 & E2 & E3 & E4 & E5 & E6 & $\begin{array}{c}\text { Concentration } \\
\text { minimale* }\end{array}$ \\
\hline Oxygène dissous (mg/L) & 2,33 & 0,89 & 5,41 & 4,03 & 4,41 & 3,74 & 6 \\
\hline centration minimale acceptable d'oxygène dissous pour les premiers stades biologiques (CCMRE, 1987; AEP, 1997; \\
on, 1997).
\end{tabular}

Tableau 2 : Moyennes annuelles des teneurs en métaux des eaux aux stations étudiées.

\begin{tabular}{|c|c|c|c|c|c|c|c|c|}
\hline & E1 & E2 & E3 & E4 & E5 & E6 & Moyenne & Valeur seuil ** \\
\hline $\mathrm{Fe}(\mathrm{mg} / \mathrm{L})$ & 2,89 & 2,33 & 1,29 & 9,25 & 1,61 & 3,97 & 3,56 & $300 \mu \mathrm{g} / \mathrm{L}$ \\
\hline $\mathrm{Cd}(\mathrm{mg} / \mathrm{L})$ & 0,13 & 0,09 & 0,09 & 0,12 & 0,10 & 0,10 & 0,10 & $0.017 \mu \mathrm{g} / \mathrm{L}$ \\
\hline $\mathrm{Pb}(\mathrm{mg} / \mathrm{L})$ & 3,23 & 1,80 & 2,02 & 2,6 & 2,86 & 1,58 & 2,35 & 1 à $7 \mu \mathrm{g} / \mathrm{L}$ \\
\hline $\operatorname{Hg}(\mu \mathrm{g} / \mathrm{L})$ & 3,12 & 12,52 & 6,12 & 5,17 & 1,35 & 1,67 & 4,99 & $\begin{array}{l}4 \quad \mathrm{ng} / \mathrm{L} \text { pour le mercure } \\
\text { inorganique et } 26 \mathrm{ng} / \mathrm{L} \text { pour le } \\
\text { methylmercure }\end{array}$ \\
\hline $\mathrm{Cr}(\mathrm{mg} / \mathrm{L})$ & 3,37 & 4,53 & 2,47 & 6,65 & 3,89 & 4,06 & 4,16 & $\begin{array}{l}1.0 \mu \mathrm{g} / \mathrm{L} \text { pour } \mathrm{Cr}(\mathrm{VI}) \text { et } 8.9 \mu \mathrm{g} / \mathrm{L} \\
\text { pour } \mathrm{Cr}(\mathrm{III})\end{array}$ \\
\hline
\end{tabular}

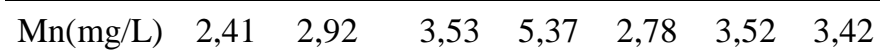

** Conseil Canadien des Ministres de l'Environnement (1999). Recommandations canadiennes pour la qualité des eaux: protection de la vie aquatique, dans Recommandations canadiennes pour la qualité de l'environnement, 1999, Winnipeg, le Conseil. 
W. CHOUTI et al. / Int. J. Biol. Chem. Sci. 4(4): 1017-1029, 2010

Tableau 3 : Matrice de corrélations entre métaux et paramètres physicochimiques.

\begin{tabular}{|c|c|c|c|c|c|c|c|c|c|c|c|c|c|c|}
\hline & $\mathrm{Fe}$ & Mn & $\mathrm{Cr}$ & $\mathbf{H g}$ & Cd & $\mathbf{P b}$ & $\mathbf{T}^{\circ} \mathbf{C}$ & pH & Cond & $\mathbf{O}_{2}$ & Turb & MES & Coul & Trans \\
\hline $\mathbf{F e}$ & 1 & 0,65 & 0,39 & $-0,02$ & $\mathbf{0 , 5 3}$ & 0,11 & 0,19 & $-0,12$ & $-0,29$ & 0,08 & 0,08 & 0,10 & 0,09 & $-0,06$ \\
\hline Mn & & 1 & 0,70 & $\mathbf{0 , 5 3}$ & 0,82 & 0,64 & $\mathbf{0 , 8 4}$ & 0,07 & $-0,64$ & 0,72 & $-0,51$ & $-0,46$ & $-0,54$ & 0,45 \\
\hline $\mathrm{Cr}$ & & & 1 & 0,10 & 0,69 & $-0,08$ & 0,08 & $-0,17$ & $-0,49$ & $-0,11$ & $-0,36$ & $-0,32$ & $-0,36$ & 0,49 \\
\hline $\mathrm{Hg}$ & & & & 1 & 0,10 & 0,14 & 0,00 & $-0,38$ & $-0,33$ & $-0,14$ & $-0,47$ & $-0,47$ & $-0,44$ & 0,27 \\
\hline Cd & & & & & 1 & 0,55 & 0,57 & $-0,11$ & $-0,53$ & 0,41 & $-0,32$ & $-0,27$ & $-0,25$ & 0,28 \\
\hline $\mathbf{P b}$ & & & & & & 1 & 0,70 & 0,21 & $-0,26$ & $\mathbf{0 , 5 2}$ & $-0,37$ & $-0,32$ & $-0,35$ & 0,09 \\
\hline $\mathbf{T}^{\circ} \mathbf{C}$ & & & & & & & 1 & 0,45 & $-0,27$ & $0, \mathbf{8 3}$ & $-0,37$ & $-0,32$ & $-0,41$ & 0,26 \\
\hline pH & & & & & & & & 1 & 0,46 & 0,75 & 0,19 & 0,23 & 0,09 & $-0,19$ \\
\hline Cond & & & & & & & & & 1 & 0,13 & $\mathbf{0 , 7 3}$ & $\mathbf{0 , 7 2}$ & 0,69 & $-0,70$ \\
\hline $\mathrm{O}_{2}$ & & & & & & & & & & 1 & $-0,12$ & $-0,08$ & $-0,17$ & 0,05 \\
\hline Turb & & & & & & & & & & & 1 & 0,99 & 0,98 & $-0,83$ \\
\hline MES & & & & & & & & & & & & 1 & 0,97 & $-0,81$ \\
\hline Coul & & & & & & & & & & & & & 1 & $-0,83$ \\
\hline Trans & & & & & & & & & & & & & & 1 \\
\hline
\end{tabular}




\section{DISCUSSION}

Les conséquences néfastes qu'engendrent les métaux sur l'écosystème aquatique ne sont plus à démontrer. La contamination des écosystèmes aquatiques par les métaux demeure un sérieux problème d'environnement de plus en plus inquiétant (Reyms-Keller et al., 1998). Ce qui a amené certains états à fixer des valeurs seuils à ne pas dépasser dans les cours d'eau afin de protéger les organismes qui y vivent. Les recommandations canadiennes pour la qualité de l'environnement, protection de la vie aquatique, qui se sont basées sur des travaux de différents chercheurs sont une preuve des précautions à prendre pour une meilleure protection des organismes aquatiques. Les valeurs moyennes obtenues lors de cette étude pour les différents métaux dépassent les valeurs seuils fixés dans ces recommandations.

En effet, au cours d'essais en écoulement continu de 60 jours, Snarski et Olson (1982) ont-ils observé une réduction de la croissance (poids) des jeunes de Pimephales promelas dont les parents avaient été exposés au mercure inorganique de concentration $0,26 \mu \mathrm{g} / \mathrm{L}$ ainsi qu'une inhibition de la reproduction prenant la forme d'une réduction du frai et de la ponte. Une gamme de concentrations mesurées de methylmercure allant de 0,04 à $0,26 \mu \mathrm{g} / \mathrm{L}$ utilisées au cours d'essais en écoulement continu a donné lieu à une diminution appréciable de la production de jeunes chez Daphnia magna (Biesinger et al., 1982). La gamme des concentrations de toxicité aiguë (24 - 96 h) du methylmercure se situe entre 24 et $125 \mu \mathrm{g} / \mathrm{L}$ pour les poissons (Thomas et Montes, 1978; Wobeser, 1975). Les concentrations de mercure mesurées au niveau de la lagune dépassent toutes les valeurs de toxicité chronique indiquées et celle du point E2 mesurée en saison sèche $(25 \mu \mathrm{g} / \mathrm{L})$ se situe dans la gamme de la toxicité aiguë. Le mercure est donc une des causes de la mauvaise qualité des eaux de la lagune et par conséquent explique en partie la mort des poissons souvent constatée par les pêcheurs.
Aussi, les mêmes remarques sont-elles faites pour le cadmium où la valeur seuil fixée par les recommandations canadiennes est de $0,017 \mu \mathrm{g} / \mathrm{L}$ et pour le chrome où la valeur seuil est de $1 \mu \mathrm{g} / \mathrm{L}$ pour le chrome hexavalent et de $8,9 \mu \mathrm{g} / \mathrm{L}$ pour le chrome trivalent. Les valeurs moyennes obtenues pour le chrome et le cadmium sur la lagune dépassent ces différentes valeurs seuils recommandées. Les eaux de la lagune sont donc de mauvaise qualité et auront pour conséquences: la diminution de la production halieutique, le retard de croissance, la disparition des espèces sensibles, la mortalité élevée des poissons. C'est cette dernière conséquence, mortalité des poissons, qui est de plus en plus remarquée au niveau de la lagune.

De plus, les teneurs de fer en saison des pluies sont-elles plus élevées qu'en saison sèche. L'une des origines du fer dans les cours d'eau étant le lessivage des sols (Didier, 1995), les eaux de ruissellement qui arrivent donc dans la lagune sont chargées de fer qui provient des déchets déposés le long de la lagune. Ces teneurs sont plus élevées au point E4 car ce point est situé non loin d'un grand dépôt de déchets d'un marché d'échanges de produits de toutes sortes.

En outre, le point E2 présente-t-il des caractéristiques physicochimiques proches de celles des eaux souterraines. En effet, la conductivité à ce point est très faible par rapport aux autres points sauf en période de hautes eaux. De même, la salinité est nulle en période d'étiage où l'apport du Lac Nokoué est le plus important. Les eaux souterraines alimentent donc suffisamment ce point, ce qui provoque une dilution, expliquant ainsi le phénomène observé.

De novembre en mars, c'est la saison sèche. L'évaporation des eaux de la lagune fait diminuer le volume d'eau, autrement dit la profondeur devient plus faible à tous les points, entrainant ainsi une concentration plus élevée des métaux analysés. C'est ce qui explique la tendance de concentration plus élevée de novembre en mars, observée pour tous les métaux en général et pour le mercure en particulier. Mais par contre, ce n'est pas le 
cas du mercure au point E2, qui au lieu d'augmenter de novembre en mars, a diminué. Ceci semble confirmer l'hypothèse d'un apport d'eaux souterraines à ce point.

La teneur en oxygène dissous est l'un des paramètres fondamentaux de la qualité de l'eau. Des études ont montré que des concentrations réduites d'oxygène entraînent des effets létaux et sublétaux (physiologiques et comportementaux) chez divers organismes, en particulier chez les poissons. Les jeunes poissons tendent à être plus sensibles que les poissons plus âgés (Alabaster et Lloyd, 1982). Une exposition à de faibles teneurs en oxygène dissous au cours du développement embryonnaire entraîne chez les embryons un retard de maturation et une hausse de la mortalité au fur et à mesure qu'ils vieillissent. En effet, l'oxygène dissous qui se trouve dans les cours d'eau sert à la respiration des organismes aquatiques. Cependant, ce phénomène n'est efficace que si l'oxygène est présent au-delà d'une certaine concentration, car les organismes aquatiques ont besoin d'une quantité minimale d'oxygène dissous pour survivre. Ainsi, la détermination de la teneur en oxygène dissous donne des indications sur la santé des cours d'eau et permet, entre autres, d'évaluer la qualité des habitats des poissons.

Les recommandations canadiennes pour la qualité des eaux correspondant aux teneurs en oxygène dissous les plus faibles qui soient acceptables sont de 6 et de $5,5 \mathrm{mg} / \mathrm{L}$ pour le stade initial et les autres stades de vie, respectivement, dans les écosystèmes d'eau chaude, ainsi que de 9,5 et de $6,5 \mathrm{mg} / \mathrm{L}$ pour le stade initial et les autres stades de vie, respectivement, dans les écosystèmes d'eau froide. Or les valeurs moyennes annuelles obtenues à tous les points sont inférieures à 5,5 mg/L. Ceux-ci ne sont que les résultats des activités anthropiques autour de la lagune. Les eaux de ruissellement, qui finissent dans la lagune, proviennent des zones de la ville où il y a présence d'installations septiques non conformes ou il n'en existe pas du tout, et les lixiviats provenant des déchets déposés le long de la lagune l'enrichissent de nutriments.
Ces eaux qui se retrouvent ainsi dans la lagune favorisent la croissance des plantes aquatiques (jacinthes d'eau) et des algues qui s'y trouvent un peu partout. Cet apport en matière organique provoque une perte nette en oxygène dissous car l'oxydation chimique directe des matières organiques nécessite de l'oxygène et par conséquent contribue à l'épuisement de l'oxygène dissous. L'oxygène disponible pour la respiration des poissons reste alors insuffisant ce qui engendre la mortalité élevée signalée par les riverains.

\section{Conclusion}

Les eaux de la lagune de Porto-Novo ne sont pas de qualité à protéger la vie aquatique. La mort des poissons souvent constatée au niveau de la lagune peut se justifier par les concentrations élevées des éléments traces métalliques étudiés et de l'appauvrissement de la lagune en oxygène dissous. Les résultats obtenus pour le fer, le manganèse et le chrome montrent que les eaux de ruissellement constituent une source non négligeable de pollution.

Le niveau de pollution de la lagune montre l'urgence de trouver des solutions adéquates pour la stopper: construire une station d'épuration, mieux gérer les déchets, sensibiliser les populations et mettre en pratique le principe du pollueur-payeur. $\mathrm{Ce}$ qui éviterait la mortalité élevée des ressources halieutiques et permettrait de préserver la santé publique.

\section{REFERENCES}

AEP (Alberta Environmental Protection). 1997. Alberta water quality guideline for the protection of freshwater aquatic life: Dissolved oxygen. Alberta Environmental Protection, Standards and Guidelines Branch, Edmonton.

Alabaster J, Lloyd R. 1982. Water quality criteria for fresh water fish. 2e éd. Food and Agriculture Organization, Nations Unies. Butterworths.

Biesinger K, Anderson E, Eaton G. 1982. Chronic effects of inorganic and organic mercury on Daphnia magna: toxicity, 
accumulation, and loss. Arch. Environ. Contam. Toxicol., 11: 769-774.

Bloundi M. 2005. Etude géochimique de la lagune de Nador (Maroc oriental): Impacts des facteurs anthropiques. Thèse de Doctorat, Université Louis Pasteur, p. 1.

Boucheseiche C, Cremille E, Pelte T, Pojer K, 2002. Guide technique $\mathrm{n}^{\circ} 7$, pollution toxique et écotoxicologie : notion de base. Lyon, Agence de l'eau Rhône Méditerranée - Corse.

CCMRE (Conseil canadien des ministres des ressources et de l'environnement). 1987. Recommandations pour la qualité des eaux au Canada. Préparées par le Groupe de travail sur les recommandations pour la qualité des eaux.

Conseil canadien des ministres de l'environnement. 1999.

Recommandations canadiennes pour la qualité des eaux : protection de la vie aquatique. dans Recommandations canadiennes pour la qualité de l'environnement, Winnipeg, le Conseil.

Didier G, 1995. La Pollution des Milieux Aquatiques: aide mémoire, 2ème édition, 220p.

Evaluation des progrès du développement durable en Afrique depuis Rio. Conférence préparatoire africaine pour le sommet mondial du développement durable, Nairobi, 15-18 octobre 2001.
Lamizana-Diallo M, Kenfach S, MillogoRasolodimby J. 2008. Evaluation de la qualité physico-chimique de l'eau d'un cours d'eau temporaire du Burkina FasoLe cas de Massili dans le Kadiogo. Sud Sciences et Tehnologies, 16: 23-28.

Rayms-Keller A, Olson E, McGaw M, Oray C, Carlson O an Beaty B. 1998. Effects of heavy metals on Aedes aegypti (Diptera : Culicidae) larvae. Ecotoxicol. Environ. Saf., 39: 41-47.

Snarski V, Olson G. 1982. Chronic toxicity and bioaccumulation of mercuric chloride in the fathead minnow (Pimephales promelas). Aquat. Toxicol., 2: 143-156.

Langstone W, Burt G, Pope N. 1999. Bioavailability of metals in sediments of the Dogger Bank (central North Sea): A mesocosm study. Estuarine, Coastal and Shelf Science, 48: 519-540.

Truelson RL. 1997. Water quality criteria for dissolved oxygen. Préparé pour le British Columbia's Ministry of Environment, Lands and Parks, Water Management Branch, Victoria. 